\title{
CONFORMATION CHANGES IN rRNA INDUCED BY ELECTRIC IMPULSES
}

\author{
Arnold REVZIN* and Eberhard NEUMANN \\ Max-Planck-Institut für biophysikalische Chemie, D-34 Göttingen, GFR \\ Received 26 March 1974 \\ Revised manuscript received 6 June 1974
}

\begin{abstract}
We have investigated the effects of electric field pulses (of about $44 \mathrm{kV} / \mathrm{cm}$ magnitude and $30 \mu \mathrm{sec}$ duration) on E. coli ribosomal RNA in solution $\left(5 \times 10^{-4} \mathrm{M}\right.$ sodium cacodylate buffer, $\left.\mathrm{pH} 7,20^{\circ} \mathrm{C}\right)$. The time course of changes in ultraviolet absorbance and electric dichroism can be resolved into contributions from orientational and conformational changes. The fast part of the relaxation spectrum (completed in less than $1 \mu \mathrm{s}$ ) is attributed to alignment of double-helical segments of $\mathrm{r}$ RNA with the external electric field; the slower part (relaxation times in the ms and $\mu \mathrm{s}$ range) is shown to be due to changes in orientation of the entire rRNA molecule, concomitant with electric field-indured helix-coil transitions of oligomeric base-paired regions.
\end{abstract}

\section{Introduction}

The analysis of electiic field-induced changes in electrical and optical properties of anisotropic polyelectrolytes [1] has yielded basic information about macromolecular size and shape, and about the relationship between the polyion and its surrounding cloud of counterions. In particular, some unusual electric birefringence data and dielectric measurements led to speculation that the polyelectrolyte ionic atmosphere might be responsible for the observed effects $\{2,3\}$. It remained for Eigen and Schwarz, using ac conductivity measurements [4], to demonstrate convincingly that rod-like polyions may be oriented by interaction of the applied electric field with dipoles induced through displacement of the counterion atmosphere parallel to the long axis of the moleculc. Indecd, numerous clectrical and electrooptical studies on polyclectrolytes can be consistently interpreted in terms of such ion-atmosphere polarization (cf., e.g., refs. [4-11]), and theoreties have been developed to account quantitatively for the phenomena $[12-15]$.

Recently it has been demonstrated that electric

* Present address: Institute of Molecular Biology, University of Oregon, Eugenc, Oregon 97403, USA. impulses cause conformational changes in macromolecules. Schwarz and Seeling [16] have presented evidence that electric fields may bring about helix-coil transitions in polypeptides (in nonaqueous solutions). Long-lived conformational changes induced in the multi-stranded polynucleotide complex poly(rA)-2 poly $(\mathrm{rU})$ by electric impulses have been analysed in terms of shifts in the counterion cloud causing strand repulsion and unwinding of helical structures [17] . As part of investigations on metastable secondary structures in macromolecules [18] we have studied the behavior of ribosomal RNA in the presence of electric fields. Previous work on electric birefringence and dichroism of rRNA has been aimed at characterizing the molecular configuration of $r$ RNA in solution $[19,20]$. In this study we present direct evidence that electric fields may cause not only changes in orientation of rRNA but also helix-coil transition in double-helical sections of the polyelectrolyte.

\section{Materials and methods}

The rRNA was a mixture of $16 \mathrm{~S}$ and $23 \mathrm{~S}$ species from Escherichia coli (strain MRE 600). Preparation and handling of RNA and of buffer solutions has been previously described [21]. 


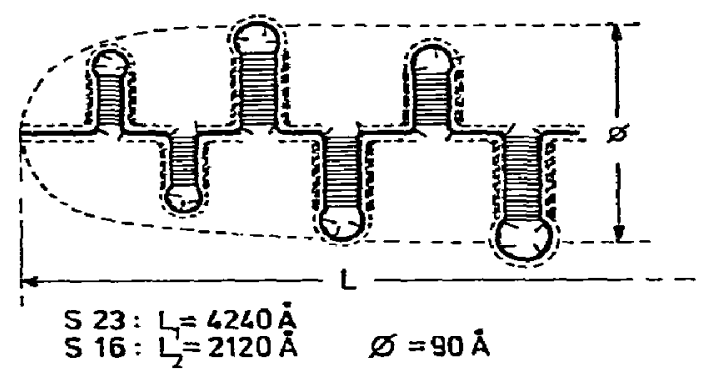

Fig. 1. Model for rRNA at pH 7 (after Spirin [241). Lengths of major axis, $L$, for $16 \mathrm{~S}$ and $23 \mathrm{~S}$ particles and of minor axis, $\phi$, were calculated under the following assumptions: (i) the 23S (16S) particle contains about 3550 (1775) bases, of which 2500 (1250) are in double-helical regions and 1050 (525) are in single-stranded stretches. (ii) At the low ionic strength used $\left(5 \times 10^{-4} \mathrm{M}\right)$ the rRNA is rather extended, and the average distance between partially stacked bases in singlestranded regions is taken to be about $4 \mathrm{~A}$. (iii) The doublehelical "arms" contain on average 15 base pairs. The distance between base gairs is taken to be 3 A [ $A^{\prime}-R N A ; S$. Arnott, Progr. Biophys. Mol. Biol. 21 (1970) 2651. Thus, the average length of an arm is about $45 \mathrm{~A}$, and its diameter is about $20 \mathrm{~A}$.

The electric impulse experiments were performed with a high power pulse generator equipped with a fast spectrophotometric detection system $[22,23]$ and polarization optics [23]. This apparatus permits easy measurement of the time course of field-induced changes in several optical properties of the sample solution.

The design of the measuring cell permits application of high field intensities while minimizing temperature increase [17]. Two flat, gold covered electrodes form the base planes of the cylindrical volume $(\approx 0.4 \mathrm{ml}$ ) of a thermostated Teflon cell. The light beam of the detection system crosses the cell through quartz windows, perpendicular to the direction of the applied electric field. When the device is used in its polarizing mode, the light may be polarized at any angle to the applied electric field [23].

\section{Theoretical aspects}

According to the generally accepted model, rRNA at neutral $\mathrm{pH}$ is a single polymeric chain in which coil regions alternate with double-helical stretches ("arms") containing about 4-20 base pairs (of the WatsonCrick type). About 70\% of the bases are in base-paired regions even at rather low ionic strengths [20]. (For review see ref. [24].) We may assume that the helical portions align approximately perpendicular to the long axis of the stretched molecule (fig. 1), especially for the case of the high electrostatic repulsion which is present at low ionic strength (e.g. $5 \times 10^{-4} \mathrm{M}$ ).

\subsection{Relaxation times}

When polyelectrolytes are subjected to an external electric field a rapid polarization is induced by displacement of the counterion cloud. The time constant for this process in rRNA under our experimental conditions may be estimated to be $\& 1 \mu \mathrm{s}$. (See, e.g., eqs. 11-14 of ref. [17]; calculations using these equations are based on Mandel's model [13] for polarization of rod-like polyelectrolytes, and account for saturation effects and dependencies on counterion type and neutral salt concentration.) Thus, when a field is applied to an rRNA solution a set of dipoles rapidly develops. Since there is some rotational freedom about sugar-phosphate bonds, the double-helical "arms" will tend to align along the direction of the applied field (fig. 2b). Concomitantly there will be rotation of the entire mactomolecule into the field direction because of the overall induced dipole moment (fig. 2c) [20] -

When the field is removed, the system relaxes to its resting state (fig. 2a). Time constants, $\tau$, for rotational relaxation of arms and of the entire molecule may be estimated from the equation $\tau=\left(6 D_{\mathrm{r}}\right)^{-1}$ (see ref. [2]) using Gans' formula for the rotational diffusion coefficient, $D_{r}$, for non-charged ellipsoids of revolution in the absence of intermolecular interactions [25].

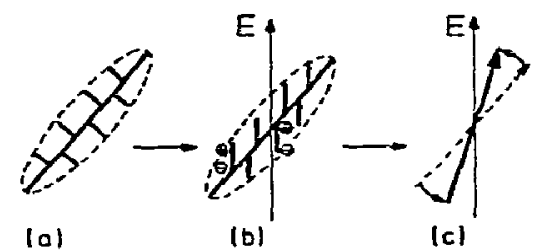

Fig. 2. Some effects of applied electric frelds on rRNA.

(a) Schematic diagram of configuration in absence of field.

(b) Applying field results in dipole formation (indicated by \pm signs on two of the "arms") and in fast alignment of "arms" with the field.

(c) Overall dipole moment causes slower rotation of entire molecule into field direction. 
$\tau=\frac{\pi \eta}{18 k T} \frac{L^{3}}{[\ln (2 L / \phi)-0.5]}$.

In eq. (1), $\eta$ is the solution viscosity, $k$ the Boltzmann constant, $T$ the absolute temperature, $L$ is the major axis and $\phi$ the minor axis of the ellipsoid. Using for average dimensions $L=45 \AA, \phi=20 \AA$, we calculate for the arms $\tau(\mathrm{arm}) \approx 0.01 \mu \mathrm{s}$. With the dimensions given in fig. 1 we estimate $\tau(165) \approx 120 \mu \mathrm{s}, \tau(23 \mathrm{~S}) \approx$ $820 \mu$ s for rotational relaxation of the entire molecules $16 S$ and $23 S$ rRNA. Thus the arms relax much faster than the molecules can rotate.

Study of multistranded polynucleotide complexes [17] suggests that the applied field may cause helixcoil transitions of base pairs in the double-helical arms of IRNA. It is also conceivable that there may occur field-induced single-strand destacking of bases although so far there is no evidence in the literature for such effects. In any case, the time constant for stacking-destacking processes in single-stranded polynucleotides may be estimated from studies on oligoand polyriboadenylates [26] to be about $0.5 \mu \mathrm{s}$.

As mentioned above, ion-atmosphere polarization may induce dipole moments in the arms of rRNA. At the negative end of such a dipole double-helical section, counterion screening of the negatively charged phosphate residues is reduced and base pairs can open. When the field is switched off and the ion cloud rapidly relaxes there will be a large driving force favouring base pair closure.

Assuming that $n$ base pairs are involved in an intramolecular "all-or-none" coil-helix transition [27], the time constant for this process is given by

$\tau_{n}^{-1}=k_{n}^{\mathrm{a}}+k_{n}^{\mathrm{d}}=k_{n}^{\mathrm{a}}\left(1+1 / \alpha s^{n}\right)$,

where $k_{n}^{2}$ and $k_{n}^{\mathrm{d}}$ are the (overall) rate constants for the association and dissociation of $\boldsymbol{n}$ base pairs, respectively, $s$ is the mean stability constant for a base pair adjacent to a base pair sequence, and $\alpha$ is a mean nucleation parameter. When the driving force strongly favors helix formation, i.e., $s \gg 1$, eq. (2) reduces to

$\tau_{n}^{-1}=k_{n}^{\mathrm{a}}$

-If a nucleus is already present (i.e. formally $\alpha=1$ ), $k_{n}^{a}$ is the rate constant for the association of $n$ base pairs adjacent to a pre-existing nucleus. Denoting by $k^{a}$ the association rate constant of a single base pair (adjacent to a nucleus), we may write for small $n$ $(\leqslant 10)$,

$k_{n}^{\mathrm{a}} \leqslant k^{\mathrm{a}} / \boldsymbol{n}$

Using eqs. (3) and (4) we thus may estimate the maximum number of base pairs which may be associated with a measured $\tau_{n}$.

In rRNA, $k^{\mathbf{a}}$ is an average over $A \cdot U$ and $G \cdot C$ base pairs. However, no direct data are available for $k_{n}^{\mathbf{a}}$ or $k^{3}$ at the low ionic strength of $5 \times 10^{-4} \mathrm{M}\left[\mathrm{Na}^{+}\right]$ we have used. Kinetic studies of helix-coil transitions in hetero-oligonucleotides with $n=6$ to 10 [28] show that the recombination rate constants $\left(k_{n}^{\mathrm{a}}\right)$ decrease with decreasing ionic strength. It seems reasonable that $k^{\mathrm{a}}$ values will be lower at low ionic strength because of higher repulsion between charged polynucleotide backbones. On the other hand, the oligonucleotide studies [28] show that the helix dissociation rates are independent of ionic strength; this implies that $k^{\mathbf{a}}$ is also independent of ionic strength and that the observed ionic strength dependence of $k_{n}^{\mathbf{a}}$ lies solely in the increased difficulty of nucleation at low salt concentrations. Thus it is not yet established whether the $k^{3}$ value of $10^{7} \mathrm{~s}^{-1}$ reported by Pörschke and Eigen [29] for oligo (rA) - oligo (rU) helixes at $0.05 \mathrm{M}$ ionic strength will apply to our system, or whether a $k^{a}$ of, say, $10^{5} \mathrm{~s}^{-1}$ is more appropriate for our ionic conditions.

\subsection{Rotational and conformational contributions to dichroism}

If we assume that the overall polarizability of rRNA is symmetric with respect to the long axis (L, fig. 1), it follows from eq. 13 of ref. [30] that $A_{\mathbb{1}}^{\mathrm{rot}}-A_{0}=-2\left(A_{1}^{\mathrm{rot}}-A_{0}\right)=\Delta A_{1}^{\mathrm{rot}}=-2 \Delta A_{1}^{\mathrm{rot}}$,

where $A_{0}$ is the isotropic absorbance (in the absence of an applied field) and $A_{1}^{\text {rot }}, A_{1}^{\text {rot }}$ are the absorbances of light polarized parallel and perpendicular to the field direction, respectively, when the molecules are rotated by the external field. When conformational changes, $\Delta A{ }^{\infty 0 n f}$, as well as rotational effects, $\wedge A^{\text {rot }}$, occur, we may write for the measured rotal absorbance changes

$\Delta A_{\mathrm{l}}=\Delta A_{\mathrm{i}}^{\mathrm{onf}}+\Delta A_{\mathrm{I}}^{\mathrm{rot}}, \quad \Delta A_{\perp}=\Delta A_{\perp}^{\mathrm{conf}}+\Delta A_{\perp}^{\mathrm{rot}}$.

Since the conformational contribution is isotropic, 
$\Delta A_{1}^{\text {conf }}=\Delta A_{1}^{\text {conf }}=\Delta A^{\text {conf }}$.

Using eqs. (6) and (8) we may solve eqs. (7) for the conformational and rotational contributions to the electric dichroism:

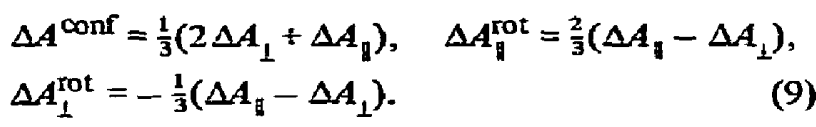

\section{Results and discussion}

\section{I. Electric birefringence}

We have used the usual optical arrangement for measurement of electric birefringence, with polarizers, on either side of the cell, rotated to $45^{\circ}$ with respect to the field direction. We find that the light intensity emerging through crossed polarizers is increased in the presence of the field while the intensity emerging through parallel polarizers is decreased. Due to the small signal amplitudes, the experimental arrangement does not produce birefringence curves which can be analyzed in detail, but the results establish that we are indeed observing a field-induced effect and not an artefact from, for example, cell distortion.

\subsection{Electric dichroism}

Fig. 3 shows the result of a typical electric dichroism experiment. The time course of each intensity change, $\Delta I$, is seen to be rather complex. There are rapid changes when the rectangular pulse starts at $t_{0}$ and when it ends at $t_{e}$; the changes during and after the pulse reflect slower processes. The long time values $(t \geqslant 10 \mathrm{~ms})$ for $\Delta I_{1}$ and $\Delta I_{1}$ are small, indicating that contributions to destacking and base-pair opening due to temperature increas $2 \approx 1^{\circ} \mathrm{C}$ under the given conditions) are negligible when compared with field-induced intensity changes.

The time constants for the fast processes at $t_{0}$ and $t_{e}$ are $\leqslant 1 \mu \mathrm{s}$. We note also that the contribution of these fast processes to $I_{I}$ is about twice as large and is of opposite sign to the contribution to $I_{1}$-According to the theoretical considerations discussed in section 3.2 , we may attribute the fast changes to (partial) rotation of double-helical arms into the field direction. This interpretation is consistent with the obser-

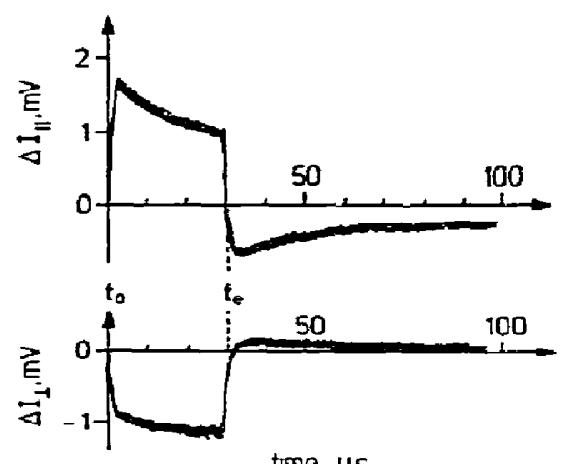

time. HS

Fig. 3. Electric dichroism data for rRNA at $20^{\circ} \mathrm{C}, 5 \times 10^{-4} \mathrm{M}$ $\mathrm{Na}^{4}$-cacodylate, pH 7; rRNA concentration of $2 \times 10^{-4} \mathrm{M}$ phosphate residues. $A 44 \mathrm{kV} / \mathrm{cm}$ pulse was applied for $30 \mathrm{\mu s}$. The impulse begins at $t_{0}$ and ends at $r_{\mathrm{e}}$. The data shown were transcribed from the actual photograph. $\Delta I_{\square}$ and $\Delta I_{\perp}$ are inteasity changes at $280 \mathrm{~nm}$ for light polarized parallel and perpendiculas to the ficld direction, respectively.

vation thai $I_{\|}$increases when the arms are being aligned. As in DNA, the transition moments of the $\pi$-electron systems of the bases are roughly perpendicular to the helix axis of the arm, which means that $I_{\|}$will grow (and $I_{\perp}$ diminish) as the arms line up along the field. It is also seen that when the field is switched off at $t_{c}$, the fast change is largely reversed, as the arms relax quickly to their original configuration. This anal$y$ sis of the fast changes is in agreement with the interpretation of electric birefringence data on rRiNA by Golub and Nazarenko [20] .

To facilitate further analysis of our dichroism results, we formally delete the amplitudes of the rapid changes (due to arm rotation) at $t_{0}$ and $t_{\mathrm{e}}$. For instance, for the data in the upper part of fig. 3 , the fast intensity change of about $1.6 \mathrm{mV}$ (completed in a negligible time after the field is applied) is subtracted from each value of $\Delta I_{\mathrm{l}}$. The slow intensity decrease from $t_{0}$ to $t_{\mathrm{e}}$ is thus referred to a value of $\Delta I_{4}=0$ at $t=t_{0}$. Likewise, the

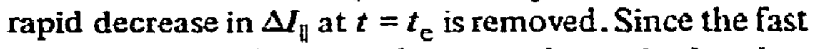
changes at $t_{0}$ and $t_{c}$ have about equal magnitudes, the initial value of $\Delta \Omega_{\mathbb{l}}$ for the slow, field-free, intensity increase coincides with the corrected final $\left(t=t_{\mathrm{e}}\right)$ value of $\Delta I_{\mathbb{l}}$ for the slow intensity decrease in the presence of the field. The slow changes thus separated out were converted to absorbance changes and are shown (schematically) in fig. 4 . (Since $\Delta I \ll I_{0}$. $I_{0}$ being the light intensity in the absence of the external field, we may approximate $\Delta A=-\Delta V / I_{0}^{\prime}$, where $I_{0}^{\prime}=2.3 I_{0}$, and 


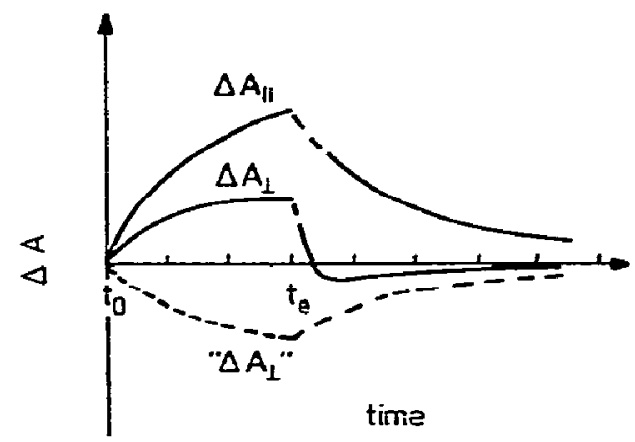

Fig. 4. Time course of electric dichroism in rRNA; the fast changes at $t_{0}$ and $t_{\mathrm{e}}$ have been deleted. (See fig. 3 and text.) Upper curves, $\Delta A_{\|}$and $\Delta A_{1}$, (schematically) represent the observed absorbance data. The lower dotted line is the curve which $\Delta A_{\perp}$ would follow if freld-induced optical changes were purely rotational.

$I_{0}=24.5 \mathrm{mV}$ ). The fact that both $\Delta A_{\sharp}$ and $\Delta A_{1}$ have the same sign shows immediately that the slower changes are not merely orientational. Were the changes purely rotational, the relationship $\Delta A_{\Downarrow}=-2 \Delta A_{\perp}$ would hold. Therefore, there must be conformational changes in addition to orientation. For the experiment shown in fig. 3 we find the absorbance changes (neglecting arm rotation) at $t=t_{\mathrm{e}}$ to be $\Delta A_{\mathrm{f}}=0.7$ $\mathrm{mV} / I_{0}^{\prime}$ and $\Delta A_{\perp}=0: 3 \mathrm{mV} / I_{0}^{\prime}$. Referring the different contributions to the sum $\Delta A_{1}+\Delta A_{\perp}=1.0 / I_{0}^{*}$ and using eqs. (9) we derive the magnitudes of the relative rotational and conformational contributions: rel. $\Delta A$ conf $=\Delta A^{\text {conf }} /\left(\Delta A_{\|}+\Delta A_{1}\right)=0.43$, rel. $\Delta A_{\sharp}^{\text {rot }}=0.27$ and rel. $\Delta A_{\perp}^{\text {rot }}=-0.135$. It is seen that the field-induced conformational changes provide a large fraction of the overall absorbance changes. From the negative value for $\Delta A_{1}^{\text {rot }}$ we infer that as the RNA molecules rotate into alignment with the field the helix axes of the double-frelical arms rotate out of the field. This implies that the limit of arm alignment is predominantly steric and electrostatic, rather than being due to randomizing tendencies of thermal motion.

\subsection{Absorbance}

Figure 5 presents typical absorbance versus time data for an clectric field pulse experiment. The changes, $\Delta A$, in such a measurement are larger than are the changes in $A_{\|}$or $A_{1}$, so we use the absorbance data for kinetic analysis. Since the time constants of proc-

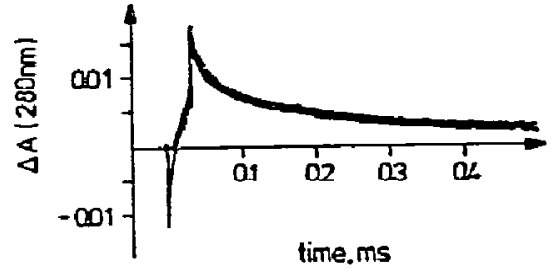

Fig. 5. Field-induced absorbance change, $\triangle A$, for rRNA as a function of time. Experimental conditions as in fig. 3.

esses involving electric polarization are dependent on the field intensity in a complex manner [4], we limit the analysis of our absorbance data, $\Delta A(t)$, to the ("field-free") time range $t \geqslant t_{\mathrm{e}}$. In fig. 6 the logarithm of the absorbance changes of a series of impulse experiments is plotted as a function time. The curvature of this plot shows that, as expected, rRNA displays a spectrum of relaxation times. Thus, we may write for $t \geqslant t_{\mathrm{e}}$,

$\Delta A(t) / \Delta A\left(t_{\mathrm{e}}\right)=\sum_{i} \beta_{i} \exp \left(-t / \tau_{i}\right)$

where $\beta_{i}$ is the relative amplitude of process $i$ having

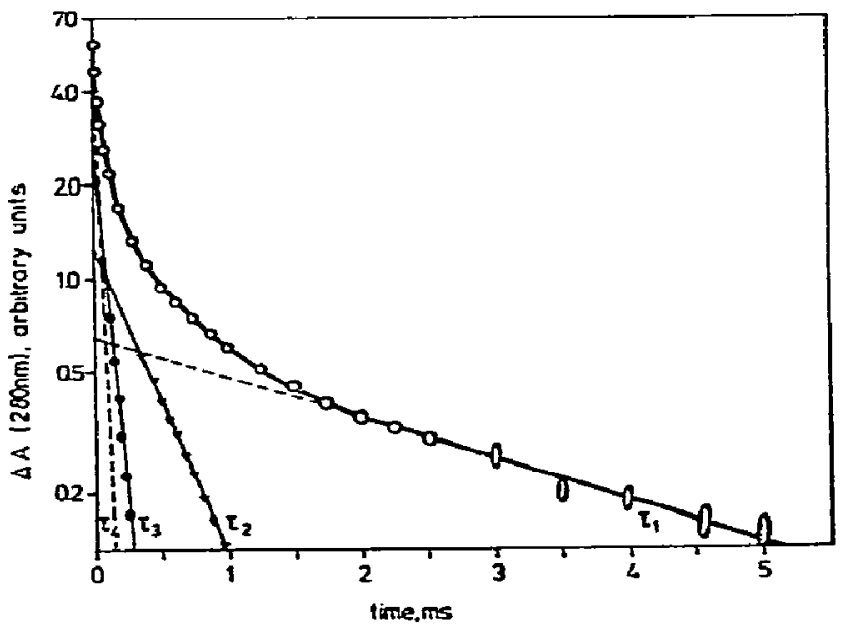

Fig. 6. Kinetic analysis of absorbance data, $\triangle A(t)$ (open circles), for $t \geq t_{c} ; \Delta A(t)$ is the difference between the absorbance value at time $r\left(\geqslant t_{\mathrm{e}}\right)$ and the value at $t \rightarrow \infty$. The initial slope of the $\log \triangle A(t)$ versus $t$ curve gives the limit, $\pi 4$, for the relaxation of the fastest process of the relaxation spectrum. 
the relaxation time $\tau_{i}[31]$. Such a spectrum may be decomposed by starting with the slowest process (characterized by $\tau_{1}$ ), subtracting contributions from this process to permit evaluation of $\tau_{2}$, etc. This approach shows that we may associate at least four time constants with the relaxation spectrum. The values obtained are: $\tau_{1}=3200 \mu \mathrm{s}, \tau_{2}=460 \mu \mathrm{s}, \tau_{3}=80 \mu \mathrm{s}$ and $\tau_{4}=30( \pm 10) \mu \mathrm{s} ; \tau_{4}$ is determined from the initial slope of the relaxation spectrum.

An analysis of this type, in which the relaxation times differ by factors of only 6 or 7 can give "meaningful" $\tau$ values only if the relative amplitudes, $\beta_{i}$, are approximately equal [32]. Extrapolation of the straight lines used to evaluate the $\tau_{i}$ to the time at which the field is switched off shows that $\beta_{1} \approx 0.10, \beta_{2} \approx 0.10$ and $\beta_{3} \approx 0.18$. Thus our analysis of the relaxation spectrum fulfils the requirement of proper amplitude ratios. Finally, the relative contribution from processes having $\tau<\tau_{3}$ is given by $\beta_{4} \approx 0.62$.

The $\beta_{i}$ values also give insight into the actual processes to which the $\pi_{i}$ may correspond. The total amplitude for the faster processes is about four times larger than that for the two slower processes. Since our experimental arrangement is such that $\Delta A \approx \Delta A_{\mathbb{Q}}$ $+\Delta A_{\perp}$, it follows from eq. (7) that

$\Delta A \approx 2 \Delta A^{\mathrm{conf}}+\frac{1}{2} \Delta A_{\mathrm{i}}^{\mathrm{rot}}$.

Using the values for $\Delta A^{\text {conf }}$ and $\Delta A_{1}^{\text {rot }}$ calculated in section 4.2 , we find that the conformational contribution to the absorbance change at $t=t_{\mathrm{e}}$ (neglecting the fast arm orientation) should be roughly six times larger than the rotational contribution. Comparing this with the $\beta_{i}$ values implies that the conformational changes are asscciated with the time range between the two smaller $\tau_{i}$ values, $\tau_{3}$ and $\tau_{4}$; while rotational effects are characterized by the two larger $\tau_{i}$ values $\tau_{1}$ and $\tau_{2}$.

This interpretation is consistent with the actual values of the $\tau_{i}$. If $\tau_{1}$ corresponds to overall rotation of 23S RNA and $\tau_{2}$ to 16S RNA, we see that the measured values of these quantities are somewhat larger than those estimated by eq. (1). However, the theoretical values of $\tau$ are very sensitive to the choice of the molecular dimension $L$; errors in the calculations may partially cancel if we consider the ratio of $r$-laxation times. This ratio is $3200 / 460=7.0$ for measured $T$ values and is found to be 6.8 from the theoretical calculation. Thus $\tau_{1}$ and $\tau_{2}$ may indeed reflect rotation of the entire RNA molecules. This analysis is also in agreement with the results of electric birefringence studies on IRNA where time constants in the msec range have been associated with macromolecular orientation [20].

Finally, the fast part of the relaxation spectrum characterized by $\tau_{4}=30 \mu \mathrm{s}$ and $\tau_{3}=80 \mu \mathrm{s}$ is clearly associated with conformational changes and probably reflects coil-helix transitions. Since most of the fast changes due to arm hending $\left[\pi(\operatorname{arm}) \ll \tau_{4}\right.$ or $\tau_{3}$ ] are reverted immediately upon switching off the field, we infer that the arms are not grossly changed by the field, i.e., the extent of helix-coil transition caused by the field is not very large.

If in fact $k^{\mathrm{a}}=10^{5} \mathrm{~s}^{-1}$, then using the values of $\tau_{4}$ and $\tau_{3}$ in eqs. (3) and (4) we find that the field pulse causes opening of $3-8$ base pairs. This would imply that few arms are totally unwound by the field. On the other hand, if $k^{a}$ is much larger than $10^{5} \mathrm{~s}^{-1}$, our data may reflect a rate-limiting nucleation step in arm refolding. The $\tau_{3}$ and $\tau_{4}$ values reported here are similar to those measured for double-helical tRNA fragments [33] (after rough corrections have been made for different ionic conditions and for different values of $s$ ). In any case, our results indicate that the conformational changes induced by the electric field involve base pair separation of short stretches in the arms of rRNA.

\section{Concluding remarks}

The orientation of macromolecules by electric fields is often used to deduce information about molecular size and shape. The present study of rRNA indicates that such electro-optical studies may be complicated by conformational changes which occur in addition to orientation, especially for long impulses of high field intensity in solutions of low ionic strength. Conversely, possible orientation of molecules should be considered when analyzing optical changes in temperature-jump experiments, since a large electric field is present as the capacitor is discharged during the jump [31] . The various contributions to field-induced changes in optical parameters may be separable by analysis similar to that used here for rRNA electric dichroism results. 


\section{Acknowledgement}

We thank: H.H. Grünhagen for generous help in using the electric impulse apparatus, D. Porrschke for critical comments, and the Stiftung Volkswagenwerk for a grant to E.N.

\section{References}

[1] E. Frcdericq and C. Heussier, Electric dichroism and electric birefringence (Clarendon Press, Oxford, 1973).

\{2\} C.T. O'konski and B.H. Zimm, Science 111 (1950) 113.

[3] H.M. Dintzis, J.L. Oncley and R.M. Fuoss, Proc. Natl. Acad. Sci. USA 40 (1954) 62.

14] M. Eigen and G. Schwarz, Z. Physik. Chem. N.F. 4 (1955) 380 ; J. Coll. Sci. 12 (1957) 181 ; in: Electrolytes, ed. B. Pesce (Pergamon Press, Oxford, 1962) p. 309.

[5] C.T. O'Konski and A.J. Haltner, J. Am. Chem. Soc. 79 (1957) 5634 ;

C.T. O'Konski and S. Krause, J. Phys. Chem. 74 (1970) 3243.

16] N. Ise, M. Eigen and G. Schwarz, Biopolymers 1 (1963) 343.

[7] S.Z. Jakabhazy and S.W. Fleming, Biopolymers 4 (1966) 793.

[8] C. Hominck and G. Weill, Biopölymers 10 (1971) 2345.

[9] D.-W. Ding, R. Rill and K.E. van Holde, Biopolymers 11 (1972) 2109 .

[10! F. van der Touw, J.W.H. Briedé and M. Mande], Biopolymers 12 (1973) 111.

[1 1 | E. Charney and J.B. Milstien, Biopolymers, in press.

[12] G. Schwarz, Z. Physik 145 (1956) 563.

[13] M. Mandel, Mol. Physics 4 (1961) 489.
[14] J.P. McTague and J.H. Gibbs, J. Chem. Phys. 44 (1966) 4295.

[15] F. Oosawa, Biopolymers 9 (1970) 677; Polyelectrolytes (Marcel Dekker, New York, 1971).

[16] G. Schwarz and J. Seelig, Biopolymers 6 (1968) 1263.

[17] E. Neumana and A. Katchalsky, Proc. Natl. Acad. Sci. USA 69 (1972) 993 .

[18] E. Neumann, Angew. Chem. 85 (1973) 430; Angew. Chem. Intern. Edit. 12 (1973) 356.

[19] G.A. Dvorkin and A.S. Spirin, Dokl. Akack. Nauk SSSR 135 (1960) 987.

[20] E.I. Golub and V.G. Nazarenko, Biophys. J. 7 (1967) 13.

[21] A. Revzin, E. Neumann and A. Katchalsky, J. Mol. Biol. 79 (1973) 95.

[22] G. Ilgenfritz, Ph.D. Thesis, Göttingen (1966).

[23] H.H. Grünliagen, Biophysik 10 (1973) 347; Ph.D. Thesis, Braunschweig (1974); Messtechnik, in press.

[24] A.S. Spirin and L.P. Gavrilova, The ribosome (Springer Verlag, Berlin, 1969).

[25] R. Gans, Ann. Physik 86 (1928) 652.

[26] D. Pōrschke, Eur. J. Biochem. 39 (1973) 117.

[27] M. Ejgen, in: Nobel Symp. Vol. 5, ed. S. Claesson (Almquist and Wiksell, Stockholm, 1967) p. 333.

[28] D. Pörschke, O.C. Uhlenbeck and F.H. Martin, Biopolymers 12 (1973) 1313.

[29] D. Pōrschke and M. Eigen, J. Mol. Biol. 62 (I971) 361.

[30| W. Kuhn, H. Dürkop and H: Martin, Z. Physik. Chem. B45 (1940) 121 .

[31] M. Eigen and L. De Maeyer, in: Techniques of organic chemistry, Vol. 8 part 2, eds. S.L. Friess, E.S. Lewis and A. Weissberger (John Wiley, New York, 1963) p. 895 .

[321 H. Strehlow and J. Jen, Chem. Instrum. 3 (1971) 47.

[33] D. Riesuer, R. Rōner and G. Maass, Eur. I. Biochem. is (1970) 85 . 